\title{
The Designing and Realization of Digital Interactive Media Art based on Activity Theory
}

\author{
Hyunil C. Kim $^{1}$ and Changyong Jung ${ }^{*}$ \\ The graduate school of Advanced Imaging Science, Multimedia \& Film, Chung- \\ Ang University, 221 Huksuk_dong, Dongjak-ku, Seoul 156-756, Korea \\ Xyrom9@gmail.com,mywood@naver.com
}

\begin{abstract}
Activity theory presents a broad and conceptual framework of analysis for HCI (Human Computer Interaction). As media art matures and becomes accepted as an art form, a theoretical and conceptual framework for its analysis and evatuation is needed. Activity theory studies the level of connection between the individuct. dommunity and society in a broad sense, and the connection's process of development is studied through different human practices as a philosophical base. As a branch of siructuralism, activity theory analyzes the connotations of meaning in human activity and not the activity itself. Activity theory comes to researchers of interactivity, intuitively. The reason for this is that activity theory provides a common langudge in comprehending the delivery of meaning from an interactive art work, in the cultural and societal sense. In order to understand the activity of the participant of the artwork through activity theory is to clarify the purpose of the activity involved, pinpoint the elements of influences in the activity systems; the artist/designer can thoroughy plan the interactive art elements, and ultimately comprehend the apprehension of the participants. The pragmatics of the system transcend its uses into artist meaning and message through the participant own history of cultural and societal experiences.
\end{abstract}

Keywords: Activity Theory, nteractive Media Art, Projection Mapping

\section{Introduction}

Computer-based interactive art is an amalgam of art and technology. The meaning of the work evolves from both its visual and experiential aspects. The interactive art experience is not only a psychological response, but constituted through exchanges that occur materially between a person and an artifact [1]. As a relatively new and amorphous genre of art, the criticism and study of it has been multi-directional as any other digitalrelated field. Human interactions that use media are mediated human interactions. Everything a human does to or with a computer is a human -computer interaction.[2] As $\mathrm{HCI}$ and media art have been cross-pollinating each other to create, experiment and develop new ideas. Filtering interactive media art through Activity theory can give researchers, theorists and artists new perspectives on advancing technologies, its influence and the involvement and evaluation, interactive art possesses and requires. Digital interactive art, which involves an interface of digital technology, and the artistic genre has been related to HCI. Applying theories of Human Computer Interaction to interactive art opens up new possibilities when planning or designing an interactive media art piece. Activity theory can provide conceptual access to a broad framework to describing the structure, development and context of computer-supported activities [3]; in interactive

* Corresponding Author 
artwork digital elements, most likely a computer-involved elements, mediate the interaction between the art work and humans. This paper does not examine the aesthetics of interactive media art but the functional component that audiences' respond to, physically react to, and together with the visual elements, ultimately derive meaning from.

\section{Activity Theory}

Activity theory was established by the Russian psychologist Vygotsky and further developed by Leont'ev, Engeström and others. Activity Theory has been applied to Human Computer Interaction as an extensive theoretical framework to analyze and evaluate it, while interactive media art has been related to HCI [4-5]. This paper directly links Activity Theory and computer-based Interactive Media Art, which specifically utilizes computer assisted interactions in order to function as an autonomous piece of art. Art viewers as participants/actors and partial-machine artworks function together to produce different dialogue for each and every viewer. Earlier HCI research and studies were based on the Cartesian ideal of cognitive science has been criticized for being unable to penetrate the human side of the interface [6]. In activity theory, an activity unit is defined with minimal context of significance as a unit of analysis. Engelström devises Vygotsky's concept of mediation as the first generation of Actixity Theory [7].

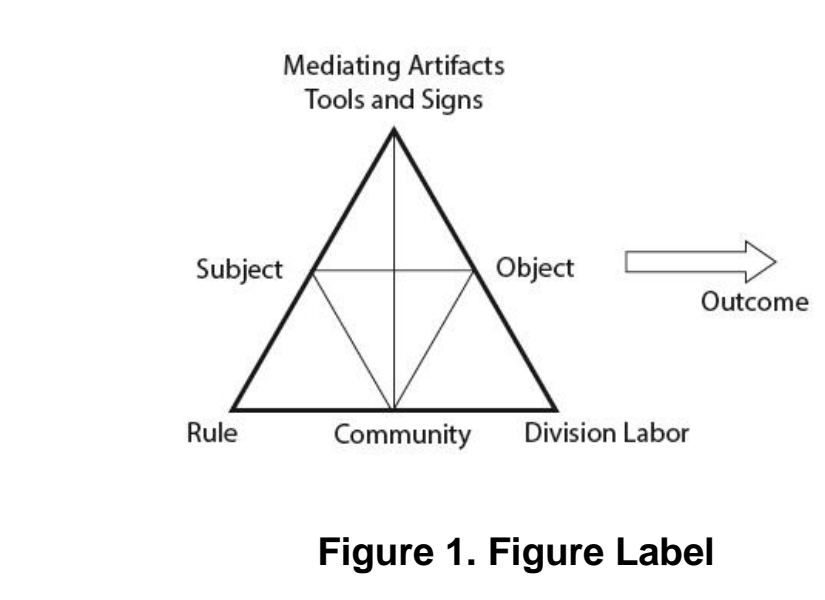

Vygotsky lays the foundations by illustrating the concept of 'a complex mediated act through a triangular model, in which the connection between the stimulus and response. His concept of cultural mediation of actions formulating a triangular structure, with the three points as subject, object and mediating artifact. This concept injects the idea of cultural aptifacts into human actions, which means objects became cultural entities and the object-orientedness of action can aid in the comprehension of human psyche. The second generation of Activity overcomes the individual focus of the analysis of activity of the first. Leont'ev explains activity within a cultural and societal context in the 2nd generation. [8] He explicates the complex relationship between individual action and a collective activity. The idea of activity advances the paradigm in that it turns the focus on complex interrelations between the community and other influences that come with an individual subject in a community (Figure1). Engelström devises a third model which illustrates where the context is accounted for, as for the collective research (Figure2). 


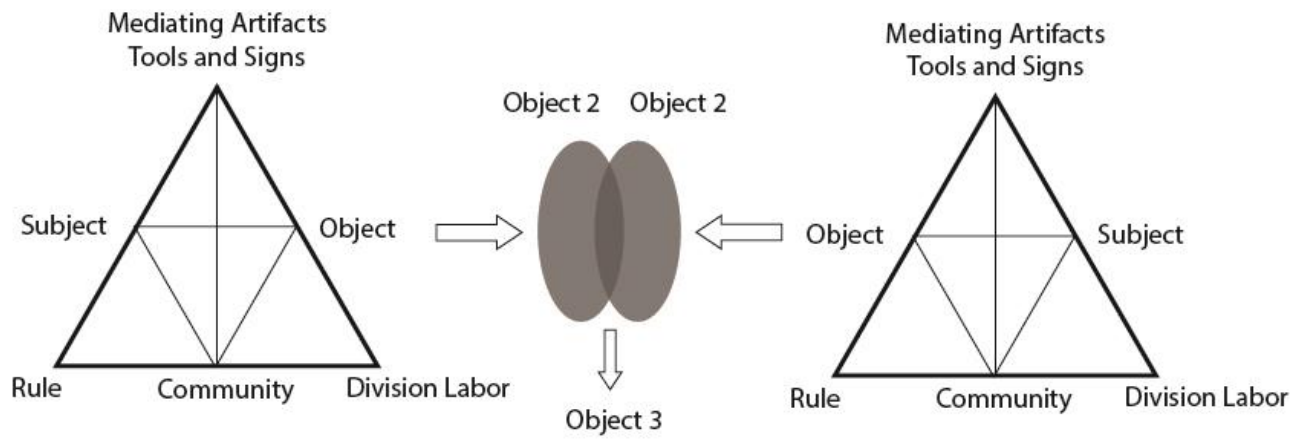

Figure 2. Figure Label

Engeström's 3rd generation activity theory model illustrates his idea of how he sees joint activity or practice as the unit of analysis, opposed to individual activity. Its intention is to develop conceptual tools to comprehend dialogues, multiple perspectives, and networks of interacting activity systems. [9] According to Engeström the object progresses from an initial situation it is given (Object 1), which is transformed to an object of collective meaning consigned by the activity system (Object 2), which then becomes a conceivably shared or mutually constructed object (Objeet 3). The figure above represents a minimal of two systems as a network; For example, when hiteractive media art work is put on display two activity systems are at work to defive meaning, that is the activity system of the individual audience member and that of the artist's. Engeström establishes five principles in order to analyze interacting Getivity systems and to expand the network, which assists in the development of aninteraetive media art project.

Firstly, the analysis of the colledive, artifact-mediated and object-oriented activity system demands the Tullest consideration as a unit. The second principle is that, the activity system possesses "multi-voicedness [10]." An activity system is a composite of diverse perspectives, traditions and interests. Historicity is the third principle, while the fourth states the pivotal roles of contradictions and instability as sources of change and development. Engeströrn expounds that contradictions are structural tensions within and between activity systems, which accumulate with history. The fifth principle declares the possibilities of extensive change in activity systems.

The figure above represents a minimal of two systems as a network; interactive media art work is composed of a system of the audience member and the system of the artist, which would constitute two systems. Meaning is derived from the activity system of the audience and the activity system of the artist interacting. The two proposed distinctly individual systems clarify how artist intention and what the audience experience can be different yet share common grounds.

\section{Case studies on Digital Interactive Media Artworks}

Activity theory is an effective and clarifying descriptive tool, which can be studied when designing or planning computer-based media art. The purpose of activity theory is to understand the wholeness of consciousness and activity, and applying its principles to one's artwork would assist the artist in the delivery of his/her intent. Since the theory reiterates naturalistic studies, culture and history, it can be the basis of how an artist understands his/her participant reaction to the piece, and how they might relate to the piece. The activity involved with the artwork is not only psychological, but is constituted through exchanges that occur materially between a person and an artifact [11]. A computer-based interactive media artwork's interactivity is the medium of the work. The 
visual network, whether tangible or not, the technical network, and the interactive activity network interact to create meaning. The signification of the activity is derived from the contingent resources of the situation the artwork presents. Activity theory's principles help analyze the situation.

\section{Activity Theory and Digital Interactive Media Art}

Projection mapping projects are analyzed as interactive media artworks in this paper. In order to establish a better example of activity theory as a framework for designing projects, interactive media art work exhibited in public spaces are chosen to make profiles of audience members non-specific and broad, instead of a specified audience, who view artwork regularly.

\subsection{YesYesNo and Others, Night Lights (2009)}

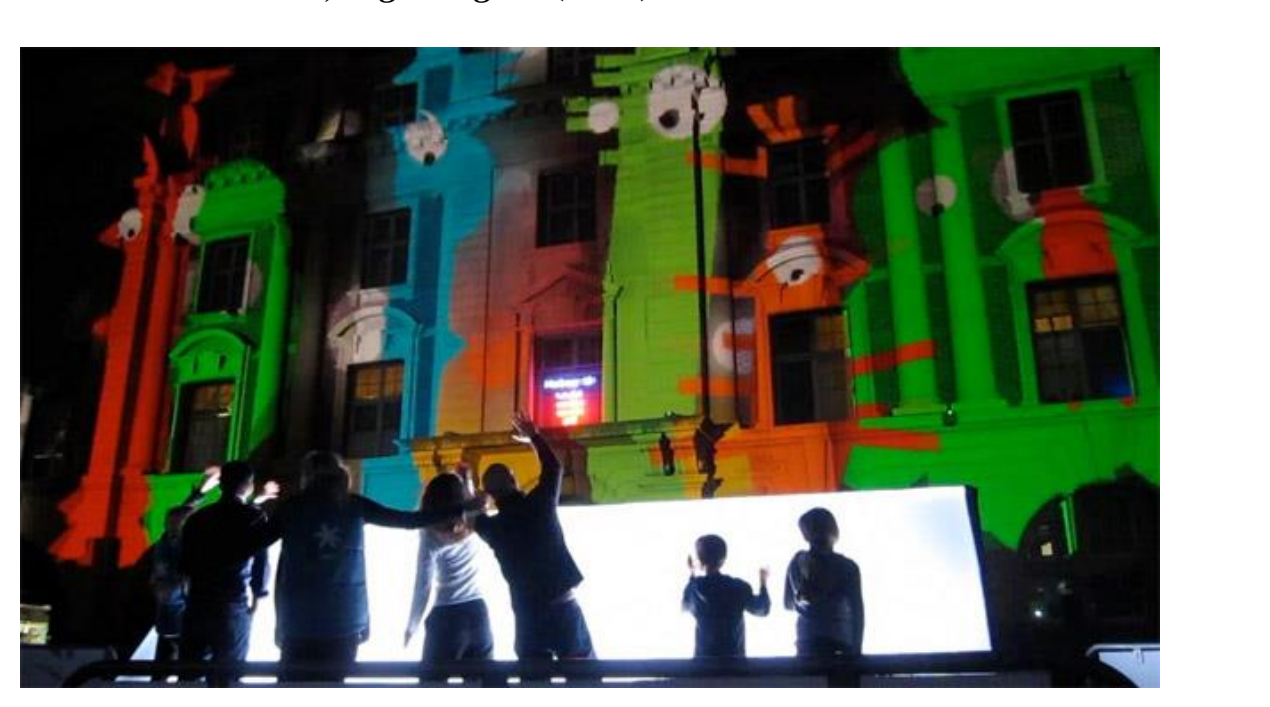

Figure 3. Figure Label

Three different types of Pnteraction interfaces were involved; 1) body interaction on two stages, 2) hand interaction above a light table, and 3) phone interaction with the tracking of waving phones. There were six scenes, cycled every hour for the public. Participants become performers in this installation. Their body movements are amplified five stories tall through projection mapping. [12] Two of the interfaces process gestures which makes it physically active piece for participants and a complex network of activity systems to analyze how the meaning is derived from its contents. 


\subsection{Architecture, Perspective lyrique (2010)}

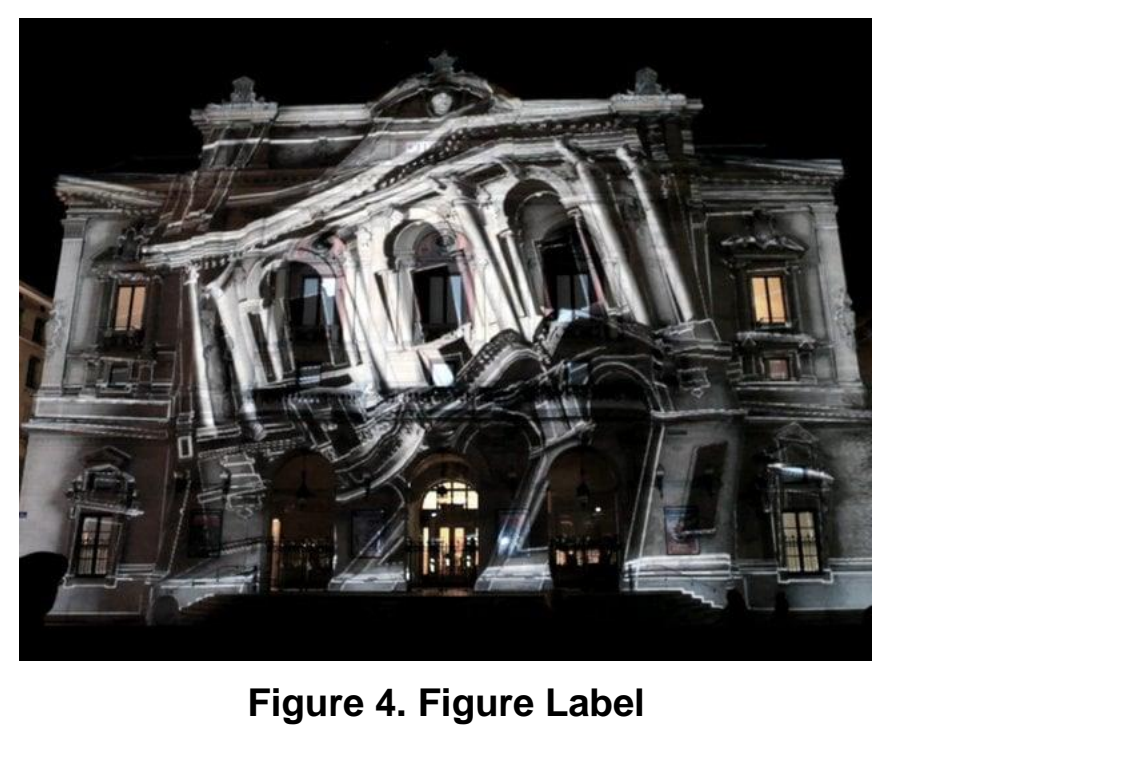

The projected building deforms and figures are controlled by the audience, using microphones and an audio analysis algorithm. This voice activated interactive interface brings participants together to make louder and different noises collectively. It also utilized a face-detection program to recognize 3 different expressions to manipulate parts of the architectural image. [13] Activity systems can be analyzed individually and collectively for this work.

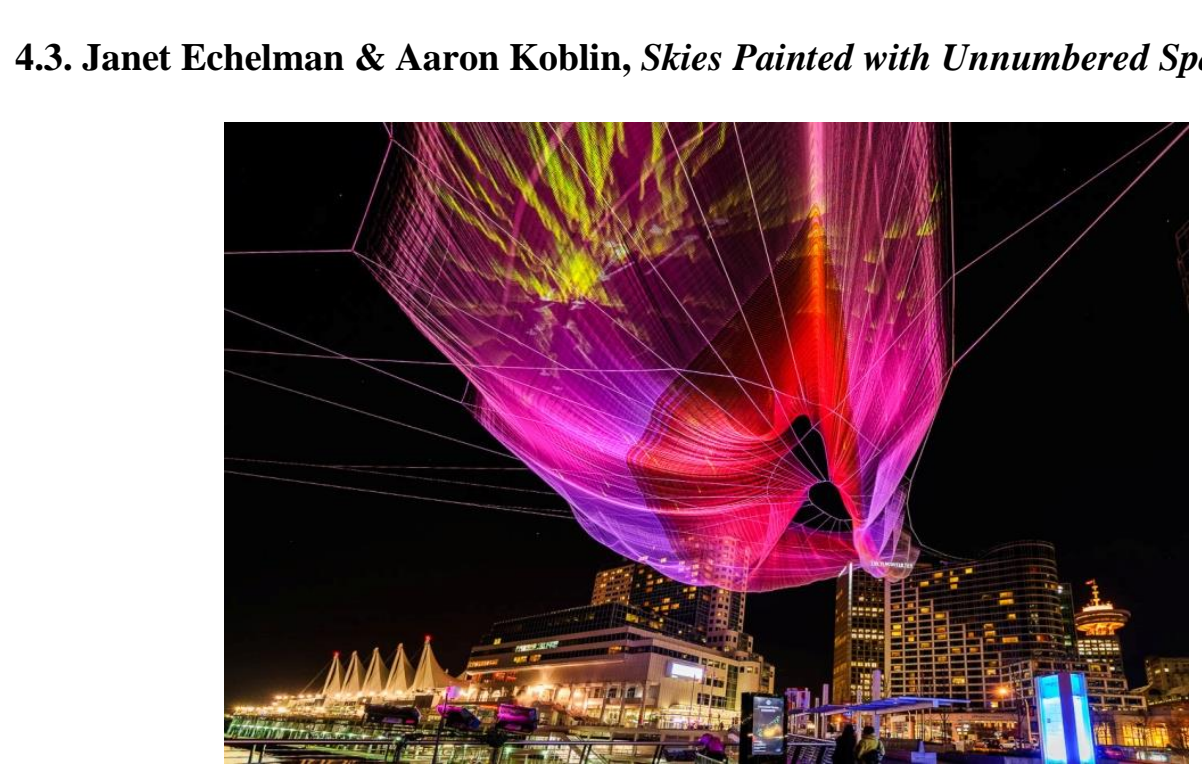

Figure 5. Figure Label

Echelman's suspended fabric sculpture at night becomes animated with interactive projection mapping. The projection is a Google Chrome web browser, displaying input from mobile devices, namely smart phone and/or tablet PCs, of participants choreographing graphical elements in real time. The complex interface consists of several innovative software's that allow personal interactions seen on mobile devices translate into a collective projection in the sky. A complexity of activity systems are at work with this piece, which is also presented as a collaboration. Sculptor Echelman and coder and 
artist Koblin have two mediating systems, which again are presented with the audience/participants activity systems to create meaning.

\section{Activity Theory of Interactive Media Artworks}

Activity systems and its principles are applied to artworks as case studies. The presented 3 interactive art pieces are analyzed by principles of activity theory's three generations. The 3 art projects present an object mediating the audience and the art work. Mobile devices, microphones and various sensors are cultural entities and objects - of which, sensors are generally implemented in a candid manner, but nonetheless cultural. These objects mediate an activity. The artist/designer/planner must design their piece to communicate their idea within this process. In other words, whether the participants are aware of their activity or not, through the individual or societal activity of interaction and participation, they should have an idea of the artists' intentions. To reiterate, the first generation of activity theory postulates that analysis of a minimal unit of individual activity to include meaning. This paper case studies, the subject. object and tools. Vygotsky(1978) explained that human activity is a process mediated through tools.

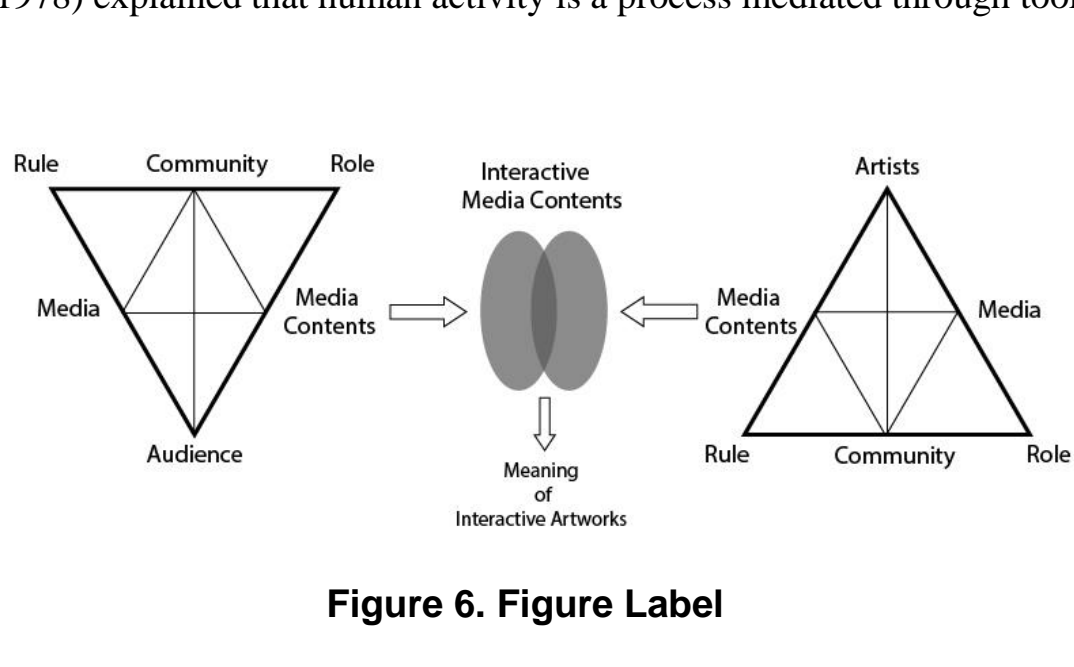

The first generation defindes activity as an act by the subject to an object, their relationship is not direct, but indirect; it is mediated through an artifact. [미주들어가야함. naver 에 있을까요?] In terms of the artifact, the subject partaking in the activity is the actor, and this is the main focus of analysis. The artifact determines the activity, its real problem and resolution which results in an activity. The activity systems suggest a resolving direction and transform the activity into a result.

Secondly the second generation of activity theory takes the first generation and put in a societal and cultural context as principle. This principle allows the investigation of the individual subject and his or her community's complex interactive relationship. The artworks of the case study have elements of operation, action and activity, which are ranked within an activity structure. Within the artworks, the participants' activities are formed by mediated artifacts (i.e. the objet, the sensor) into a result. The activities are consecutive, and the consecutive activities are manipulated. When the viewer/participants lose their motivation for action, they gain activity, and when the object of activity changes, manipulation takes place. As viewers repeat their activity, without the subject's awareness the strategy of manipulation takes place. On the other hand, if the conditions of manipulation shift, the viewer's conscience must be stimulated to form another activity.

The second principle of the activity system is about the existence of multi-voicedness, which contains multiple perspectives, tradition and interest. Multi-voicedness is a lens for the production process of the contents of interactive media art. The artist and participants, both parties bring different perspectives, tradition, and interest to the work. The artwork is produced a certain way, but meaning can be drawn from the artwork in multiple ways. 
Thirdly, an activity system has historicity. The principle speaks of how the system is formed over time, and changed in the same period. The participants bring their own prior experiences to the interactive media art, and activate the work through the work's interface. Most works do not come with a user manual. Most interfaces are designed to be intuitive, yet participants still have prior experience with interfaces and initially act based on their prior experiences. Participants will experiment with the interface and vary their activities to trigger more interactions; their initial acts from past experience feel natural, while variations of the act or new activities will change the way they interact with interfaces elsewhere.

The fourth principle by Engestrom is contradiction, and this is essential to the analysis of digital interactive media artwork. Contradiction is the source of change and progress, and the conceptual tool to analyze the interacting activity systems inherent in interactive media art. Contradiction appears when the participant interacts with the medium and intention of the computer-based media artwork. Contradiction lies between the participants activities and the interface. Various contradictions generate confusion and conflict, but functions as an innovative force of change within the activity systems of interactive media artworks. [Engestrom, 1987, 2000; Sawchuk, 2006]

The fifth and final principle is the possibility of expansive transformations. Expansive transformations occur when participants interact with an interactive media artwork, and expansively interpret the meaning of the activity object and apply and reflect the meaning in a progressive methodology. This includes new and unique meanings/objects and practices

\section{Conclusion}

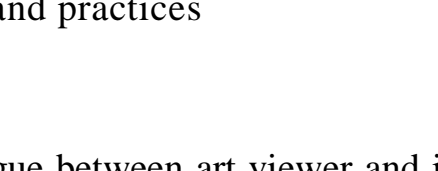

The quality of the dialogue between ant viewer and interactive art work is determined by the designing of the interface. The artist/designer implementing digital technologies should consider activity theory as a base structure to evaluate how the technology and the art works' message is interpreted and communicated. The activity of the participant has meaning suggested by the activity itself, externally and internally, and others associated from previous experiences and influences. The output of the artwork system is an activity that solidifies the message and/or deepens the meaning in addition to the sensorial response. The audience will interpret the visual and technical contents of the interaction in their own ways; the ipterpretation is contingent on what each individual brings to the artwork. They bring their own rules, histories, pre-conceived notions to the art work and participate in the art work individually. By understanding how the different systems act, react, and interact with each other, artists can realize their interactive projects by creating systems-of content to successfully deliver complex meaning.

\section{References}

[1] L. Muller, E, Edmonds, and M. Connell, "Living laboratories for interactive art", CoDesign., vol. 2, no. 4, (2006), pp. 195-207.

[2] C. Heeter, "Interactivity in the context of designed experiences", Journal of Interactive Advertising, vol. 1, no. 1, (2000), pp. 3-14.

[3] V. Kaptelinin and B. A. Nardi, "Activity theory: basic concepts and applications", CHI'97 Extended Abstracts on Human Factors in Computing Systems, ACM, (1997), pp. 158-159.

[4] L. Calvi, "Where Art and HCI meet: Towards an Art of Interaction", Proceedings of the 2nd International Symposium on Culture, Creativity, and Interaction Design, New Castle, Great Britain, (2011).

[5] D. England, "Art and interaction: some reflection", In Proceedings of the 2010 international conference on The Interaction Design, British Computer Society, (2010), pp. 46-51.

[6] K. Kuutti, "Activity theory as a potential framework for human-computer interaction research", Context and consciousness: Activity theory and human-computer interaction, (1996), pp. 17-44.

[7] Y. Engeström, "Expansive learning at work: Toward an activity theoretical reconceptualization", Journal of education and work, vol. 14, no. 1, (2001), pp. 133-156. 
[8] Y. Engeström, "Expansive learning at work: Toward an activity theoretical reconceptualization", Journal of education and work, vol. 14, no. 1, (2001), pp. 133-156.

[9] M. Cole and Y. Engeström, "A cultural-historical approach to distributed cognition", Distributed cognitions: Psychological and educational considerations, Harvard, (1993), pp. 1-46.

[10] Y. Engeström, "Expansive learning at work: Toward an activity theoretical reconceptualization", Journal of education and work, vol. 14, no. 1, (2001), pp. 133-156.

[11] L. Muller, E. Edmonds and M. Connell, "Living laboratories for interactive art", CoDesign, vol. 2, no. 4, (2006), pp. 195-207.

[12] http://www.yesyesno.com/night-lights/

[13] https://1024d.wordpress.com/category/event/perspective-lyrique/

[14] http://www.unnumberedsparks.com/

[15] P. Sawchuk, N. Duarte and M. Elhammoumi, "Critical perspectives on activity: Explorations across education, work, and everyday life", Cambridge University Press, (2006).

[16] J. Kimura and H. Shibasaki, "Recent Advances in Clinical Neurophysiology", Proceedings of the 10th International Congress of EMG and Clinical Neurophysiology, Kyoto, Japan, (1995).

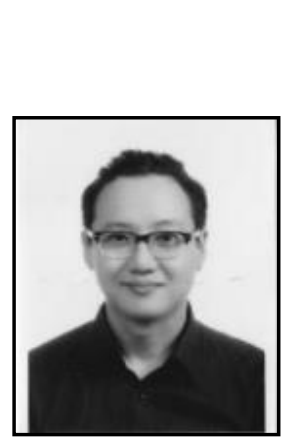

\section{Authors}

Hyunil Kim, was born in Hamilton, Ontario, Canada, He received his MFA in Studio Art from Hunter College at the City College of New York in 2007. Currently, he is pursuing his Ph.D. in Art and Technology at the Graduate School of Advanced Imaging Science, Multimedia and Film, Chang-Ang University, Seoul, Korea. He also teaches photography an various colleges. His research interests include photography, video, experimental film Theory, critical race theory, and media art

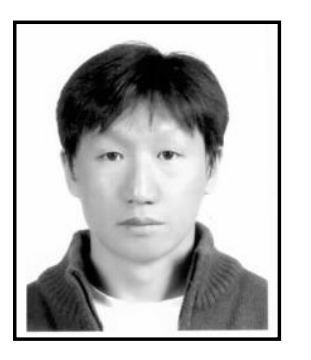

Changyong Jung, was boyn in Seoul, Korea, He received his Ph.D In Art and Technology at the Graduate School of Advanced Imaging Science, Multimedia and Film, Chung-Ang University, Seoul, Korea. Research Interests: 3D Projection mapping, Interface Design, 3D Contents, Media façade, New Media Art, Interactive media ant

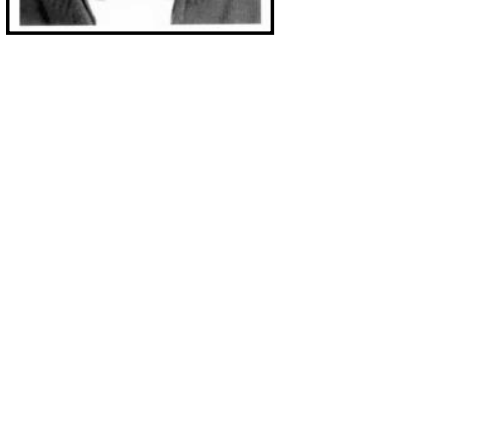

